\title{
Tcf7L2 is essential for neurogenesis in the developing mouse neocortex
}

\author{
Olga Chodelkova ${ }^{1}$, Jan Masek ${ }^{1,3}$, Vladimir Korinek', Zbynek Kozmik ${ }^{1,4}$ and Ondrej Machon ${ }^{1,2^{*}}$ (D)
}

\begin{abstract}
Generation of neurons in the embryonic neocortex is a balanced process of proliferation and differentiation of neuronal progenitor cells. Canonical Wnt signalling is crucial for expansion of radial glial cells in the ventricular zone and for differentiation of intermediate progenitors in the subventricular zone. We detected abundant expression of two transcrtiption factors mediating canonical Wnt signalling, $T \subset f 7 L 1$ and $T c f 7 L 2$, in the ventricular zone of the embryonic neocortex. Conditional knock-out analysis showed that Tcf7L2, but not Tcf7L1, is the principal Wnt mediator important for maintenance of progenitor cell identity in the ventricular zone. In the absence of Tcf7L2, the Wnt activity is reduced, ventricular zone markers Pax6 and Sox2 are downregulated and the neuroepithelial structure is severed due to the loss of apical adherens junctions. This results in decreased proliferation of radial glial cells, the reduced number of intermediate progenitors in the subventricular zone and hypoplastic forebrain. Our data show that canonical Wnt signalling, which is essential for determining the neuroepithelial character of the neocortical ventricular zone, is mediated by Tcf7L2.
\end{abstract}

Keywords: Neurogenenesis, Neocortex, Wnt signalling, Tcf7L1, Tcf7L2

\section{Introduction}

The neocortex in mouse is formed during midembryogenesis in the prosencephalon as a multi-layer structure from cortical progenitors cells [1]. Neural progenitor cells in the cortical ventricular zone (VZ) termed radial glial cells (RGC) generate neurons directly during asymmetric mode of cell division giving rise to one progenitor daughter cell and one daughter neuron, or one intermediate progenitor cell (IPC). IPCs may undergo few rounds of cell division in the subventricular zone (SVZ) before terminal differentiation into postmitotic neurons [2-4]. In the early forebrain at embryonic stages (E) 8-11 days post coitum, neural progenitors divide symmetrically to rapidly expand the progenitor pool before the asymmetric mode of cell division is initiated [5]. Several signalling pathways including Wnt are involved in regulation of neurogenesis. At E8-11, canonical $\mathrm{Wnt} / \beta$-catenin signalling promotes symmetric division in the forebrain [6-8] while later (from E12.5 onwards)

\footnotetext{
* Correspondence: machon@img.cas.cz

'Institute of Molecular Genetics of the Czech Academy of Sciences, Vídeňská 1084, 14200 Prague, Czech Republic

${ }^{2}$ Institute of Experimental Medicine of the Czech Academy of Sciences,

Vídeňská 1084, 14200 Prague, Czech Republic

Full list of author information is available at the end of the article
}

it promotes terminal differentiation of IPC into neurons [9-12]. Transition of radial glial cells to IPCs is accompanied with the loss of adherens junctions and neuroepithelial identity [3]. Canonical Wnt signaling is mediated through activation of $\beta$-catenin and its translocation to the nucleus in which $\beta$-catenin associates with transcription factors of Tcf/Lef family and regulates expression of Wnt target genes. Classical loss-of-function studies of canonical Wnt signalling employ conditional deletion of $\beta$-catenin in various tissues including the neocortex $[8,13]$. Since $\beta$-catenin is involved both in forming adherens junctions and in controlling transcription of Wnt target genes this approach is not suitable for addressing $\beta$ catenin role during neocortical development. Abrogation of canonical Wnt signalling downstream of $\beta$-catenin, however, may provide an experimental approach that does not perturb cell adhesion properties of radial glial cells. We therefore focused on the role of Tcf/Lef transcription factors in the neocortex. Although all four proteins bind to $\beta$-catenin and to identical DNA consensus binding site, Lef1 and Tcf7 (formerly named Tcf1) operate as transcriptional activators, Tcf7L1 (formerly Tcf3) acts as a repressor while Tcf7L2 (formerly Tcf4) may exhibit both functions depending on the cellular context 
$[14,15]$. Lef1 is expressed in the tip of the medial cortical wall and it is crucial for the dentate gyrus development while the lateral cortex is normal in Lef1-/- mutants [16]. $T c f 7 L 1$ and $T c f 7 L 2$ are abundantly expressed in the forebrain while $T c f 7$ is not $[17,18]$. Here we examined the expression patterns of $T c f 7 L 1$ and $T c f 7 L 2$ in the neocortex at critical neurogenic stages between E13 and E17 and analyzed neural differentiation upon conditional inactivation of TcF7L1, Tcf7L2 or both. We provide evidence that $T c f 7 L 2$ as a transcriptional activator controls the neuroepithelial character of RGC progenitor zone and RGC proliferation.

\section{Results}

Tcf7L1 and Tcf7L2 are expressed in the VZ of the lateral and medial neocortical wall during embryonic neurogenesis

Canonical Wnt activity in the mouse embryonic neocortex was mapped using BAT-Gal reporter mouse in which multiplicated Tcf consensus binding sites coupled to a minimal promoter drive the expression of the $\beta$-galactosidase gene [19]. The strongest activity was observed in the hem and the medial cortical wall that gradually declined laterally $[9,20]$. This reflects a well known signalling centre in the hem expressing a number of Wnt proteins including Wnt3a [21]. We performed in situ hybridization on sections of the neocortex from BAT-Gal mice using antisense probe for $\beta$-galactosidase mRNA. Figure 1a illustrates the medial-high to laterallow gradient of the Wnt activity in the mouse neocortex at E15. Next, we asked what Tcf/Lef factor may mediate canonical Wnt signalling in this brain region. Immunohistochemistry on cortical sections using specific antibodies revealed that Lef1 expression correlates with medial-lateral gradient of BAT-Gal reporter (Fig. 1d). This is in the line with the idea that the hippocampus and the dentate gyrus are patterned by Wnt3a signalling through Lef1-regulated gene expression [16]. Tcf7L1 was strongly expressed in the lateral neocortical $\mathrm{VZ}$ and the signal declined in the medial wall and the hem (Fig. 1b, h). Tcf7L2 was abundant in the dorsal thalamus (DT) (Fig. 1c) corresponding to strong expression of Tcf $7 L 2$ mRNA as detected by in situ hybridization [18]. Interestingly, we detected a clear presence of Tcf7L2 protein in the VZ of the neocortex and ganglionic eminences (GE) by immunohistochemistry on sections which was not previously indicated by in situ hybridization (Fig. 1c, k). Both Tcf7L1 and Tcf7L2 proteins were present in the VZ at analyzed stages from E13 to newborn stage P0 (data not shown).

\section{Conditional inactivation of TcfTL2 but not Tcf7L1 results in severe defects in the embryonic neocortex and hippocampus}

The expression of $T c f 7 L 1$ and Tcf7L2 suggested that these two factors might control the transcriptional output of canonical Wnt signalling in the neocortex. To test this assumption we employed tissue-specific deletion of these Tcf factors. We crossed forebrain-specific Cre driver mouse line D6-Cre allowing loxP-recombination in the neocortex from E11.5 [22] with conditional alleles of Tcf7L1 [23] and Tcf7L2. D6-Cre/ROSA26 lineage tracing in whole-mounts and on coronal sections of brains at E13.5, E15.5 and newborns (P0) illustrated Cre activity in the lateral and medial neocortex $(\mathrm{Cx})$ and in the hippocampus (Hi) (Fig. 1e-g). Immunohistochemical analysis of the resulting D6-Cre/Tcf7 $\mathrm{L} 1^{\mathrm{fl} /+} / \mathrm{Tcf} 7 \mathrm{~L} 2^{\mathrm{fl} / \mathrm{fl}}$ crosses showed moderately reduced levels of Tcf7L1 expression while Tcf7L2 was not detectable. In D6-Cre/ Tcf7L $1^{\mathrm{fl} / \mathrm{fl}} / \mathrm{Tcf}_{\mathrm{L}} 2^{\mathrm{fl} / \mathrm{fl}}$ double mutants, however, both proteins were absent in the $\mathrm{VZ}$ in the area of D6-Cre recombination confirming efficient inactivation of $T c f 7 L 1$ and $T c f 7 L 2$ in the neocortex at E15 (Fig. 1h-m).

The phenotypic changes upon deletion of both Tcf factors were thoroughly examined at newborn stage $\mathrm{P} 0$. The brain size in D6-Cre/Tcf7L $1^{\mathrm{fl} / \mathrm{fl}} / \mathrm{Tcf}_{\mathrm{L}} 2^{\mathrm{fl} / \mathrm{fl}}$ double mutants was smaller compared to control littermates as illustrated by the length of cortices (arrows) (Fig. 2a). Measurement of the perimeter of cortices in controls and mutants has confirmed statistically significant size reduction in single Tcf7L2 $(87.4+/-4 \%)$ and double Tcf7L1/Tcf7L2 mutants $(88.3+/-4.2 \%)$ compared to controls (Fig. 2b). On the other hand, the size difference between Tcf7L2 single and Tcf7L1/Tcf7L2 double mutants was not statistically significant ( $p$-value: $p=0$. $75)$. Hematoxylin-eosin-stained coronal sections revealed severe changes in the brain morphology (Fig. 2c- $\mathrm{f}^{\prime}$ ). Neocortical VZ and SVZ almost disappeared in double mutants (arrows in Fig. $2 \mathrm{f}-\mathrm{f}^{t}$ ). The neocortex was thinner and cortical layering was not distinct. The hippocampus was progressively reduced towards the posterior part in which it was almost absent (asterisk in Fig. $2 \mathrm{f}^{\prime}$ ). This probably reflects the already reported higher recombination rate of D6-Cre driver in the posterior forebrain [22]. We further analyzed single mutants Tcf7L1 (D6Cre/Tcf $\left.7 \mathrm{~L} 1^{\mathrm{fl} / \mathrm{fl}} / \mathrm{Tcf} 7 \mathrm{~L} 2^{\mathrm{fl} /+}\right)$ and single mutants $T c f 7 L 2$ (D6-Cre/Tcf7L $1^{+/+} / \mathrm{Tcf}_{\mathrm{L}} 2^{\mathrm{fl} / \mathrm{fl}}$ ). The phenotype in single Tcf7L2 mutants resembled the double mutant (Fig. 2e-e, arrows). In contrast, the morphology in single Tcf7L1 mutants appeared normal (Fig. 2d-d'). These data suggest that Tcf7L2 is the principal transcription factor mediating canonical Wnt signaling in the neocortex.

\section{Neuroepithelium in the neocortical VZ is disintegrated in D6-Cre/Tcf7L2 ${ }^{\mathrm{fl} / \mathrm{fl}}$ mutants and RGC proliferation is reduced}

To confirm the laminar patterning defect and the VZ disruption in the double mutants, we performed immunohistochemistry on coronal sections of brain at P0 using specific cellular markers. The number and position of Pax6-positive $\left(\mathrm{Pax6}^{+}\right)$RGC in the VZ were unaltered 


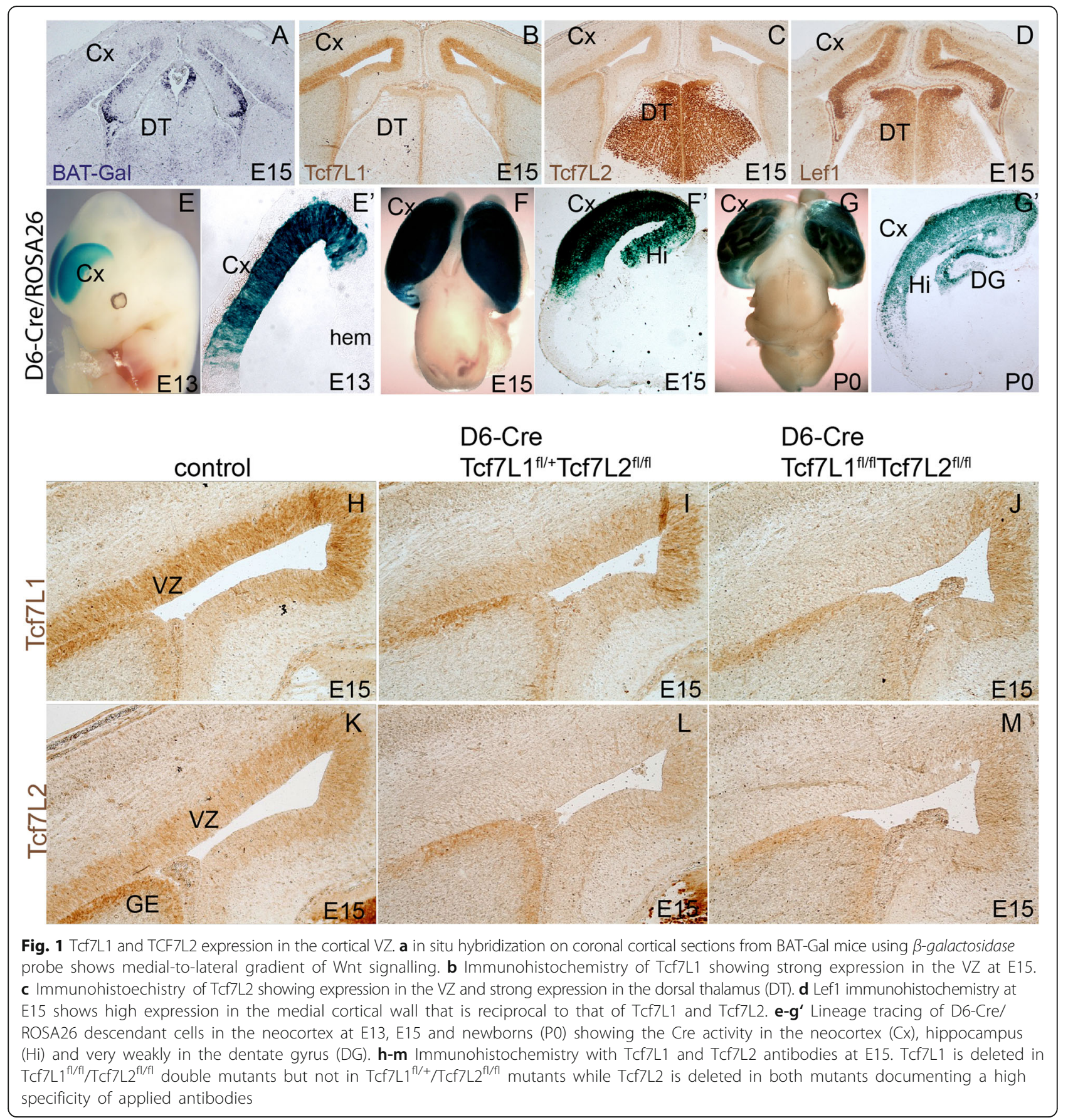

in single Tcf7L1 mutants (D6-Cre/Tcf7L $1^{\mathrm{fl} / \mathrm{fl}} / \mathrm{Tcf}_{\mathrm{L}} 2^{\mathrm{fl} /+}$ ) (Fig. 3b-b') compared to control littermates in which around $70 \%$ of all DAPI ${ }^{+}$cells were Pax6+ (Fig. 3a-a', e). In contrast, the number of Pax6 $6^{+}$RGC was reduced to one half $(35+/-3.1 \%$ of all cells) in single Tcf $7 L 2$ mutants and to $43+/-10.1 \%$ in double mutants Tcf7L1/ Tcf7L2 (Fig. 3c-d', e). Statistical analysis of $\mathrm{Pax6}^{+}$cell quantification confirmed no significant differences between controls and Tcf7L1 mutants (Student's paired t-test: $p$ value $(\mathrm{p})=0.81$ and between Tcf7L2 and
Tcf7L1/Tcf7L2 $(p=0.15)$ Fig. 3e). Next, we monitored the number and position of neurons in the cortical layers 5-3 labelled with Ctip2. Again, the number of $\mathrm{Ctip}^{+}$neurons remained unchanged in single $T c f 7 L 1$ mutants $(29+/-2.6 \%)$ compared to controls $(30.6+/-2$. $7 \%, p=0.45)$. On the other hand, single Tcf7L2 and double $T c f 7 L 1 / T c f 7 L 2$ mutants displayed significant reduction of neurons to $18.8+/-5.4 \%$ and $20.2+/-7.1 \%$, respectively, in the cortical plate (Fig. 3a", b", c", d"). Quantifications of $\mathrm{Ctip}^{+}$cells are summarized in 


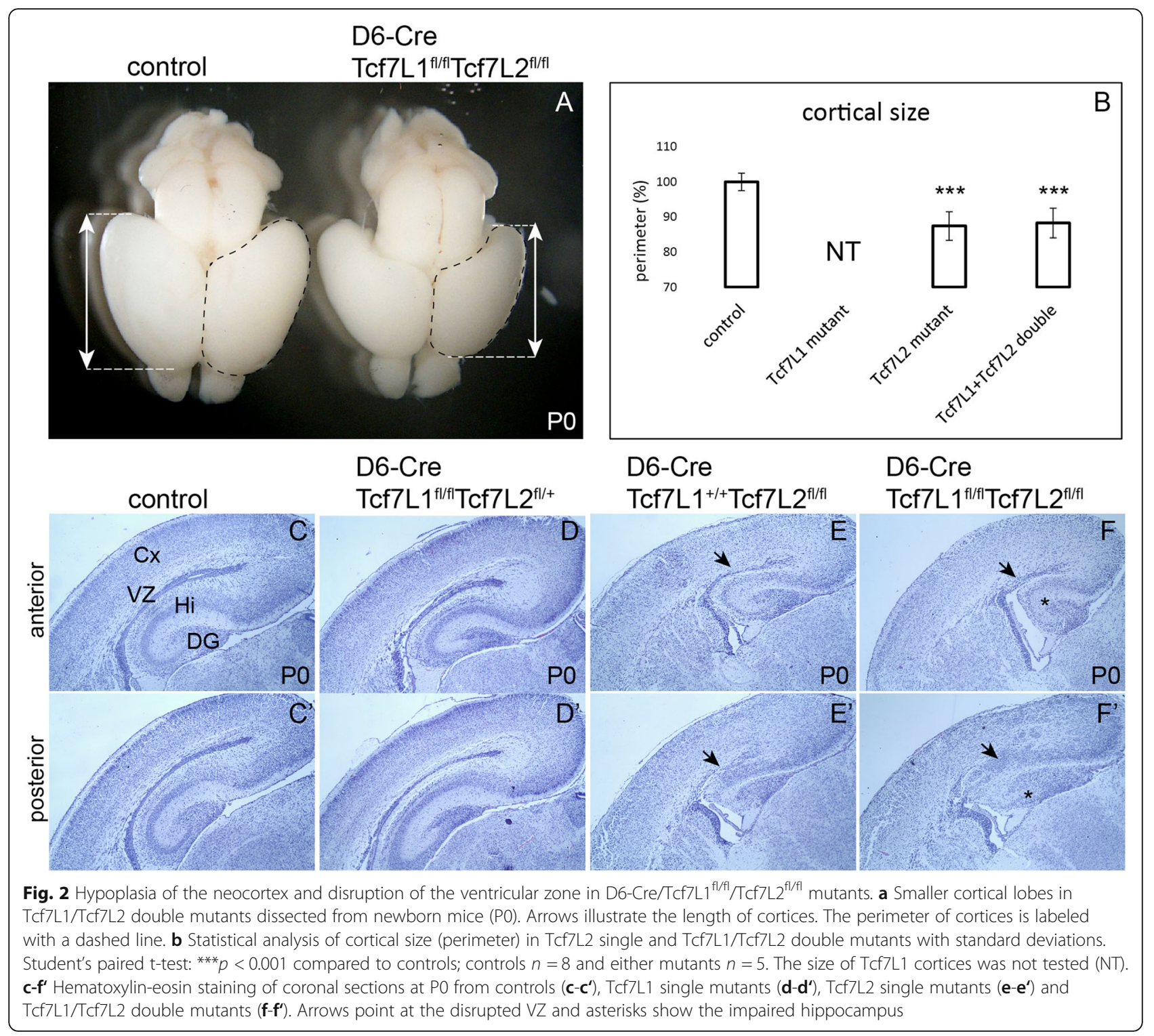

(Fig. 3f). Again, we observed no statistical significance in the number of $\mathrm{Ctip}^{+}$cortical neurons between Tcf7L2 and Tcf7L1/Tcf7L2 double mutants $(p=0.74)$. First phenotypic changes including decreased numbers of $\mathrm{Pax6}^{+}$RGC and Ctip2 ${ }^{+}$neurons were observed at E17.5 (Additional file 1: Figure S1a-h"). At E15.5, however, we did not notice any dramatical alterations in the structure and cell composition of the neocortex of D6$\mathrm{Cre} / \mathrm{Tcf} 7 \mathrm{~L} 1^{\mathrm{f} / /+} / \mathrm{Tcf}_{\mathrm{L}} 2^{\mathrm{fl} / \mathrm{fl}}$ and D6-Cre/Tcf7L1 ${ }^{\mathrm{f} / \mathrm{fl}} / \mathrm{Tcf} \mathrm{L} 2^{\mathrm{fl} / \mathrm{fl}}$ mutants as analyzed by immunofluorescence of $\mathrm{Pax}^{+}$ RGCs, Ctip2 ${ }^{+}$or Tuj $1^{+}$cortical plate, Tbr2 ${ }^{+}$IPCs in the $\mathrm{SVZ}, \mathrm{PH}^{+}$proliferating cells and $\mathrm{ZO}^{+}$adherens junctions (Additional file 2: Figure S2a-i').

Neuroepithelial progenitors in the VZ are characterized by adherens junctions at the apical side. These cellcell junctions are maintained by polarized localization of cadherins and catenins and they can be labelled with, for instance, the ZO1 marker. We checked the integrity of adherens junctions by $\mathrm{ZO} 1$ immunofluorescence in the mutants at P0 stage. As depicted in Fig. 3h', i, j', k', ZO1 labelling at the apical side was completely lost in the lateral cortical wall in single $T c f 7 L 2$ and double $T c f 7 L 1 /$ Tcf $7 L 2$ mutants compared to controls. Again, single Tcf $7 L 1$ mutants displayed normal ZO1-labelled lining of the apical side of RGC (arrow in Fig. 3i'). Next, we calculated the number of dividing cells in the VZ using immunofluorescence with PCNA antibody. In controls and single Tcf7L1 mutants, around $97 \%$ of RGC expressed PCNA marker and proliferated (Fig. 3h, i) whereas in single $T c f 7 L 2$ and double Tcf7L1/Tcf7L2 mutants, the number of dividing cells in the VZ was reduced to $20.1+/-2.9 \%$ and $15.1+/-2.6 \%$, respectively 


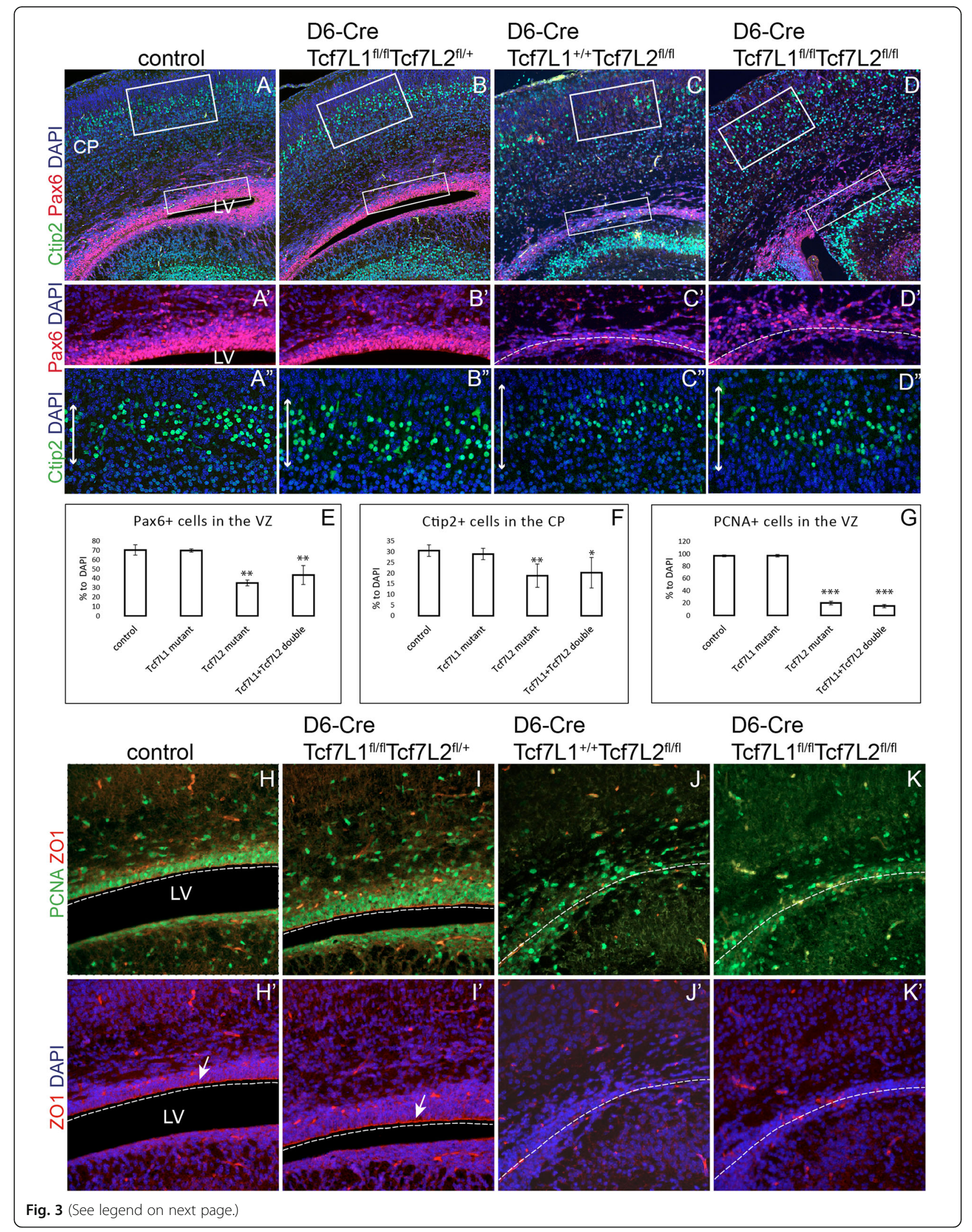


(See figure on previous page.)

Fig. 3 The neuroepithelial structure is severely impaired in the absence of Tcf7L2. $\mathbf{a}-\mathbf{d}^{\prime}$ Pax $6^{+} R G C$ and Ctip2 $2^{+}$cortical neurons are reduced upon inactivation of Tcf7L2 alone (c-c') or upon simultaneous deletion of Tcf7L1 and Tcf7L2 (d-d"). Inactivation of Tcf7L1 alone had a little influence on RGC and neuronal populations (b-b'). Panels in $\mathbf{a}^{\prime}$ and $\mathbf{a}^{\prime \prime}$ display magnified framed rectangles in $\mathbf{a}$. e-g Quantifications of Pax6 ${ }^{+}$, Ctip2 $^{+}$and $\mathrm{PCNA}^{+}$dividing cells showing an average from three independent experiments with standard deviations. Student's paired t-test: ${ }^{*} p<0.05$, ${ }^{* *} p<0.01,{ }^{* * *} p<0.001$ compared to controls, $n=8$. $\mathbf{h}-\mathbf{k}^{\boldsymbol{\prime}}$ Double immunofluorescent labelling of PCNA ${ }^{+}$dividing cells and $\mathrm{ZO} 1^{+}$apical adherens junctions in RGC. Dashed lines represent potential ventricular lining. Vertical arrows depict the thickness of the cortical layers II-IV containing Ctip2 ${ }^{+}$neurons

(Fig. 3j, k). The summary of quantifications from three independent experiments is shown in Fig. 3g. In conclusion, Tcf $7 L 2$ deletion caused severe reduction of RGC proliferation that was accompanied with disruption of the neuroepithelial structure of the VZ and neocortical stratification defects.

\section{The loss of Tcf7L2 in the embryonic neocortex leads to down-regulation of canonical Wnt signalling and reduction of radial glial cells in the VZ and intermediate progenitors in the SVZ}

Gain-of-function studies using constitutively active form of $\beta$-catenin demonstrated that proliferation of early neural progenitors is regulated by Wnt signalling [6, 24]. We therefore tested the Wnt activity upon the loss of $T c f 7 L 1$ and $T c f 7 L 2$. For monitoring in vivo, we crossed the Wnt reporter mouse line BAT-Gal [19] to the D6-Cre/ $\mathrm{Tcf} 7 \mathrm{~L} 1^{\mathrm{fl} / \mathrm{fl}} / \mathrm{Tcf}_{\mathrm{L}} 2^{\mathrm{fl} / \mathrm{fl}}$ compound strain. The expression output of Wnt signalling was measured on brain sections at P0 using immunofluorescence with an antibody against $\beta$-galactosidase. In controls, BAT-Gal ${ }^{+}$cells were localized to the VZ in which they represented around $24.1+/-2.2 \%$ of all DAPI ${ }^{+}$cells (Fig. $4 \mathrm{a}^{\mathrm{a}} \mathrm{a}^{\mathrm{a}}$ ) and the expression gradient corresponded to in situ hybridization shown in Fig. 1a. As expected, inactivation of $T c f 7 L 1$ alone had negligible, if any, effect on the Wnt activity in the neocortical VZ with $22.9+/-2.1 \%$ of BAT-Gal ${ }^{+}$cells $(p=0.55$ compared to controls) (Fig. 4b-b'). However, deletion of Tcf $7 L 2$ or both $T c f 7 L 1 / T c f 7 L 2$ resulted in decrease to $10.2+/-0.7 \%$ and to $8.1+/-1.2 \%$ of all DAPI ${ }^{+}$cells, respectively, of BAT-Gal ${ }^{+}$ cells (Fig. 4c-d'). Quantifications from three independent experiments are summarized in Fig. 4e.

Proliferation and differentiation of IPC is dependent on Wnt signalling as well $[9,20]$. We therefore immunotainstained intermediate progenitors using Tbr2 antibody in Tcf7L1/Tcf7L2-defficient cortices. Panels in Fig. 4h-k "depict double labelling of Sox $2^{+}$ RGC and $\mathrm{Tbr}^{+}$IPC progenitors in single and double Tcf mutants. The number of Sox $2^{+}$progenitors decreased from $45.6+/-0.9 \%$ in controls to $19.5+/$ $0.5 \%$ in $T c f 7 L 2$ and to $23.7+/-6.6 \%$ in $T c f 7 L 1 / T c f 7 L 2$ mutants while $T c f 7 L 1$ single mutants displayed an unchanged number of Sox $2^{+}$cells in the VZ (44.4 +/- 0.9, $p=0.2$ ) (Fig. 4f). Similarly, we observed a dramatic decrease of Tbr2 ${ }^{+}$cells in the SVZ in Tcf7L2 and Tcf7L1/
Tcf7L2 mutants from $22.6+/-3.6 \%$ to $14.6+/-3.1 \%$ and $14.0+/-1.3 \%$, respectively (Fig. $\left.4 h^{\prime \prime}-k^{\prime \prime}\right)$. Calculations from three independent experiments are shown in Fig. 4f-g. Altogether, lack of Tcf72 in the neocortical VZ leads to downregulation of canonical Wnt signalling and depletion of RGC which results in reduced IPC and hypoplasia of the neocortex.

\section{Discussion}

In this work, we identified Tcf7L2 as the principal transcription factor mediating canonical Wnt signalling in the embryonic neocortex (summarized in Fig. 5). Although $T c f 7 L 2$ is massively expressed in the dorsal thalamus its relatively weaker expression in the neocortical ventricular zone during neurogenic stages is sufficient to control transcription of Wnt target genes. Tcf7L2 has been shown to either activate or repress expression depending on the cellular context that may include the presence of other Tcf factors in the same cells, splicing variants etc. [14, 25-28]. Our experiments using BAT-Gal reporter mouse strain show that Tcf7L2 positively regulates Wnt target genes in the neocortical VZ. On the other hand, our data show that Tcf7L1 has negligible effect on neurogenesis in the mouse cortex although its expression in the $\mathrm{VZ}$ appears even stronger than that of Tcf7L2 (Fig. 1b-c). Using D6-Cre, which initiates recombination after E11.5 in the dorsal forebrain [22], deletion of Tcf7L1 alone had no effect on neurogenenis in our hands. Thus functions of Tcf7L1 and Tcf7L2 are not redundant in this area which may be explained by the fact that Tcf7L1 in most cases represses transcription [29]. This implicates that repression of Tcf targets is not necessary to set a proper transcriptional output of Wnt signalling in the cortex and that Wnt expression in the medial and lateral wall is sufficient to pattern arealization and control neurogenesis in the embryonic dorsal forebrain. The hippocampal defect in $\mathrm{D} 6-\mathrm{Cre} / \mathrm{Tcf} 7 \mathrm{~L} 1^{+/+} / \mathrm{Tcf} 7 \mathrm{~L} 2^{\mathrm{fl} / \mathrm{fl}}$ resembles severed hippocampus in $\mathrm{Lef}^{-/-}$mice $[16,17]$ indicating that hippocampus development in the medial cortical wall requires both Lef1 and Tcf7L2. Tcf7L $2^{-/-} / \mathrm{Tcf}^{-/-}$ embryos die around 14.5 with multiple defects but the forebrain morphology was not studied in detail [30] and we thus present here the first study of Tcf7L2 function in this tissue. 


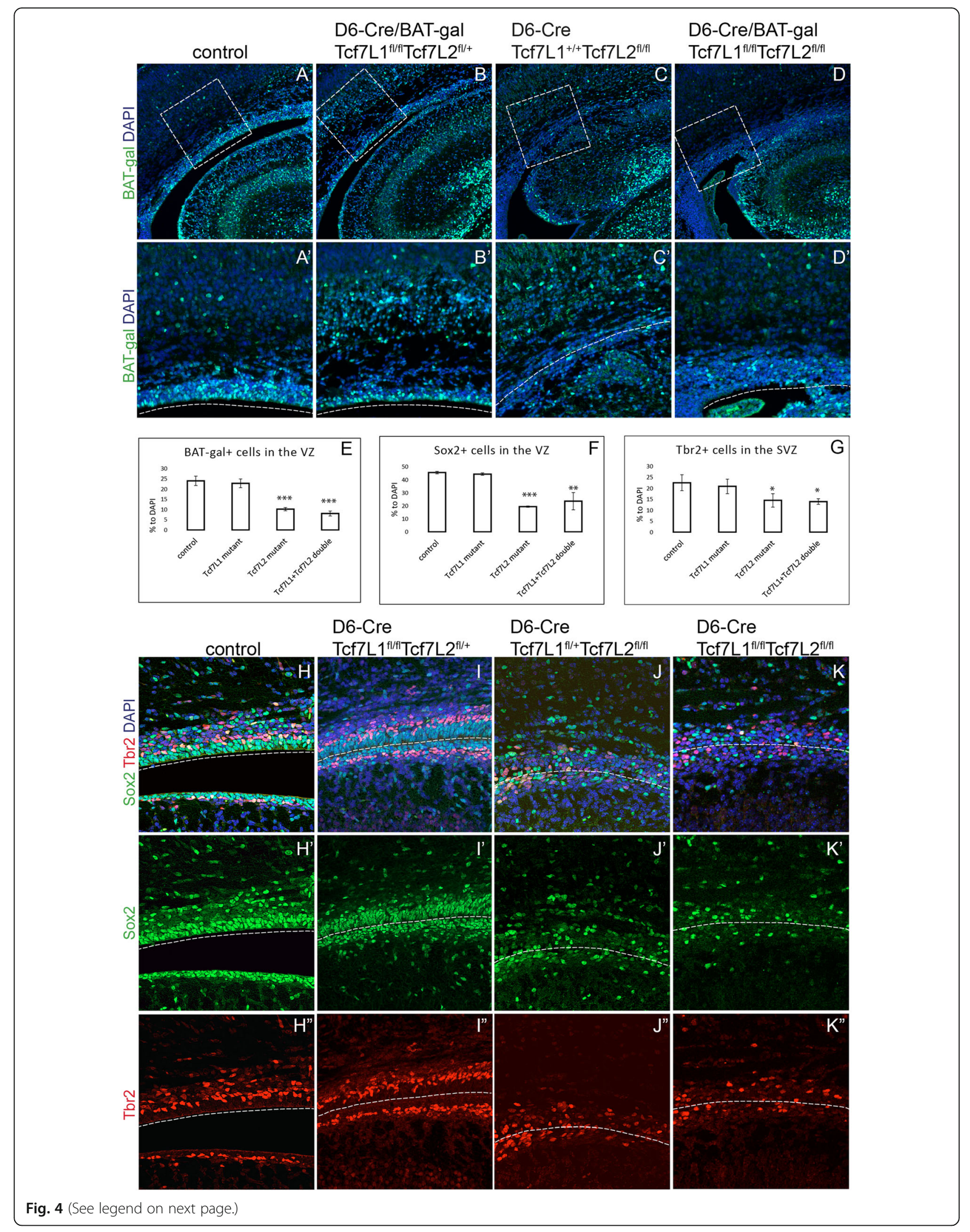


(See figure on previous page.)

Fig. 4 The loss of TCf7L2 in the embryonic cortex leads to downregualtion of canonical Wnt signalling and reduction of radial glial cells and intermediate progenitors. $\mathbf{a}-\mathbf{d}^{\prime}$ ' $\beta$-galactosidase immunofluorescence in the cortical VZ in BAT-Gal Wnt reporter mice is reduced after inactivation of Tcf7L2 alone (c-c') or upon simultaneous deletion of Tcf7L1 and Tcf7L2 (d-d'). Inactivation of Tcf7L1 alone had a little effect on BAT-Gal Wnt reporter $\left(\mathbf{b}^{-} \mathbf{b}^{\prime \prime}\right)$. Panels in $\mathbf{a}^{\prime}$ display magnified framed rectangles in $\mathbf{a} . \mathbf{e - g}$ Quantifications of BAT-Gal ${ }^{+}$, Sox $2^{+}$RGCs and Tbr2 ${ }^{+}$IPCs showing an average from three independent experiments with standard deviations. Student's paired t-test: ${ }^{*} p<0.05,{ }^{* *} p<0.01,{ }^{* *} p<0.001$ compared to controls, $n=6$. $P$-values between Tcf7L2 and Tcf7L1/Tcf7L2 mutants are: BAT-Gal ${ }^{+} p=0.06$, Sox $2^{+} p=0.34$, Tbr2 $2^{+} p=0.80$ showing no statistical significance. $\mathbf{h}-\mathbf{k}^{\prime \prime}$ Double immunofluorescent labelling of Sox2 ${ }^{+}$RGC and Tbr2 $2^{+}$IPCs. Dashed lines represent potential ventricular lining

Kuwahara and coauthors [31] reported that inhibition of Tcf7L1 by shRNA in vitro in dissociated cortical progenitors led to increased neuronal differentiation documented by increased levels of Tbr2 and $\beta$-tubulin. Further, experiments using overexpression or inhibition of Tcf7L1 by in utero electroporation of mouse cortices also concluded that Tcf7L1 is involved in maintaining RGC population by inhibiting neuronal differentiation [32]. Our genetic approach, on the other hand, did not confirm that Tcf7L1 plays a major role in cortical neurogenesis. This discrepancy could be explained that neural progenitors cultured in vitro are exposed to nonphysiological levels of Wnts in which repressor activity of overexpressed Tcf7L1 may influence neuronal differentiation.

D6-Cre/Tcf7L1/Tcf7L2 mutants display severed neuroepithelium (Figs. 2f, 3kc). $\beta$-catenin associtates with $\mathrm{N}$ cadherin and other proteins which participate in forming a polarized cytoarchitecture. Apically localized adherens junctions in radial glial cells are typical of the neuropithelium lining ventricles. Loss $\beta$-catenin or its constitutive activation leads to disruption of adherens junction and neuroepithelial integrity $[8,20]$. Thus, Wnt lossand gain-of function studies employing genetic ablation or modification of $\beta$-catenin yield complex phenotypic changes that are always accompanied with impaired adherens junction and cell-cell contacts. These defects include the loss of the neuroepithelial identity, the loss of RGC anchor in the VZ, rossette-like structures in the VZ or neuronal heterotopias [9]. In D6-Cre/Tcf7L1/ Tcf7L2 mutants, however, $\beta$-catenin stability and intracellular localization is controlled by physiologically normal levels of Wnt signalling which should not cause aberrant cell contacts. Surprisingly, we detected a complete loss of adherens junctions as a consequence of Tcf7L2 genetic deletion. This suggests that Wnt-controlled gene expression, but not direct modification of $\beta$-catenin, is critical for adherens junctions formation. It remains to be determined what adherens junction components or determinants of $\mathrm{VZ}$ progenitors are direct or indirect Wnt targets. It is possible that Sox2, which is decreased in D6-Cre/Tcf7L2 mutant VZ, is directly controlled by Tcf7L 2 as shown by binding of Tcf factors close to transcription start site of Sox2 [33].

Draganova et al. [13] used a specific conditional allele of $\beta$-catenin, Catnnb1 ${ }^{\mathrm{dm} / \text { flox }}$, for inactivation of canonical Wnt signalling in the cortex by Emx1-Cre. This Catnnb1 ${ }^{\mathrm{dm} / \text { flox }}$ variant maintained intact adherens junctions and yielded a decrease in RGC proliferation, subsequent depletion of RGC and IPC progenitor pool leading to reduced neuron number which corresponds to our data. Nevertheless, Draganova and coauthors did not detect disruption of the VZ neuroepithelium in Emx1-Cre/Catnnb1 ${ }^{\mathrm{dm} / \text { flox }}$ mutants up to E18.5, the latest analyzed stage. We report here that reduction of RGC

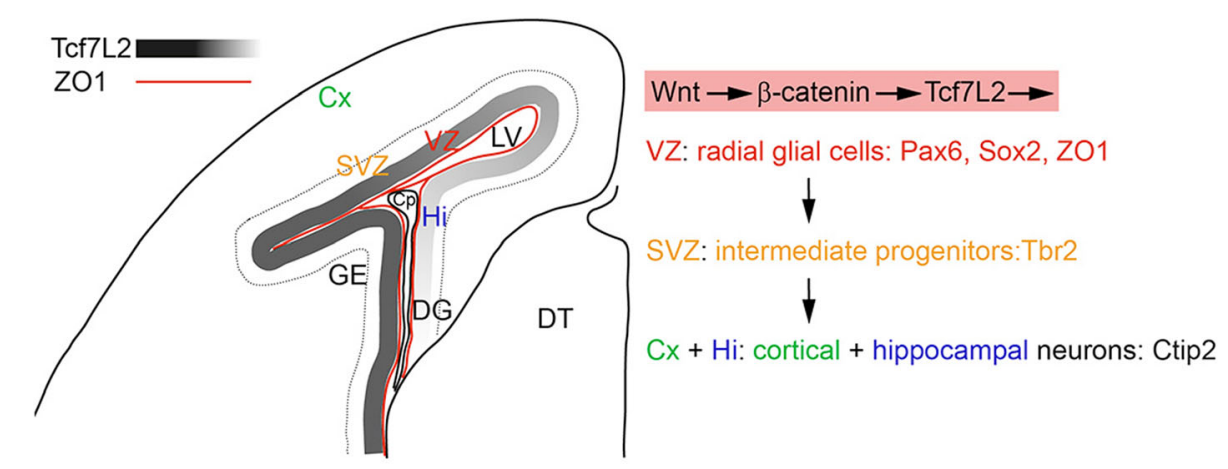

Fig. 5 Summary diagram illustrating a part of the anatomical structure of one hemisphere of the embryonic forebrain at E17. Expression gradient of Tcf7L2 in the VZ and adherens junctions lining the lateral ventricle (red line) are shown. Dashed line depicts outer boundary of SVZ. Canonical Wnt signalling through Tcf7L2 affects cell identity of radial glial cells in the VZ and their proliferation. Applied cellular markers of neuronal progenitors for distinct stages of neuronal differentiation in the cortex are listed. Cp: choroid plexus, Cx: cortex, Hi: hippocampus, DG: dentate gyrus, DT: dorsal thalamus, GE: ganglionic eminences, LV: lateral ventricle, SVZ: subventricular zone, VZ: ventricular zone 
population in D6-Cre/Tcf7L2 mutants precedes adherens junctions defect that becomes clearly visible at P0.

Although we observed efficient deletion of Tcf7L1 and Tcf7L2 at E15, the decreased number of RGC became apparent not before E17. A similar, delayed'effect of Emx1Cre/Catnnb1 $1^{\text {dm/lox }}$ deletion on cortical neurogenesis was reported by Draganova et al. [13]. We show that IPC population was clearly reduced at $\mathrm{P} 0$, probably as a secondary effect of RGC depletion. This indicates that the temporal control of Tcf7L2 target genes regulating cell cycle and identity of RGC is not absolutely dependent on active Wnt signalling.

\section{Material and methods \\ Mice}

Generation and description of these mouse strains has been described previously: D6-Cre [22], Tcf7L $1^{\mathrm{fl} / \mathrm{fl}}$ [23], ROSA26 [34]. Tcf7L2 ${ }^{\mathrm{f} / \mathrm{fl}}$ mouse strain was generated from Tcf7l2 ${ }^{\text {tmla(EUCOMM)Wtsi }}$ mice that were purchased from the European Conditional Mouse Mutagenesis Program EUCOMM (Welcome Trust Sanger Institute).

\section{Immunohistochemistry and in situ hybridization}

Immunohistochemistry was performed on paraffinembedded 5- $\mu \mathrm{M}$ thick sections using standard protocols. Primary antibodies: Lef1 (Cell Signaling 2230), Tcf7L1 (Santa Cruz, sc-8635), Tcf7L2 (Cell Signaling, 2569), Tcf7 (Tcf1, Cell Signaling 2203) Sox2 (Santa Cruz, sc17320), $\beta$-galactosidase (Abcam ab9361, ab616), Tbr2 (Abcam, ab23345), ZO-1 (Santa Cruz, sc-10804), PCNA (Sigma, P8825), PH3 (Millipore, 06-570), Tuj1 (R\&D, MAB1195). Secondary antibodies: biotinylated antirabbit or anti-mouse, Vectastain $\mathrm{ABC}$ Elite kit and ImmPACT DAB susbstrate (all Vector Laboratories). Donkey anti-rabbit, anti-mouse Alexa-Fluor 488 or 594 (Thermofisher).

In situ hybridization protocol and preparation of antisense $\beta$-galactosidase riboprobe was described in [20]. Confocal images were obtained on Leica SP5 confocal microscope. Wide-field fluorescence images were taken on Zeiss Imager.Z2.

\section{Additional file}

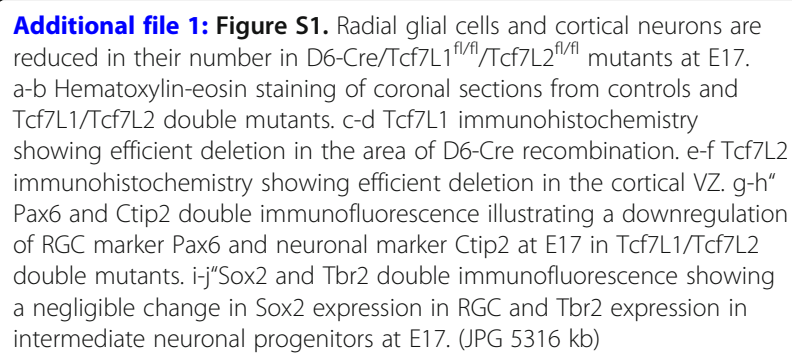

Additional file 2: Figure S2. Radial glial cells, cortical neurons and structure are not altered in in D6-Cre/Tcf $/ \mathrm{L} 1^{\mathrm{fl} / \mathrm{fl}} / \mathrm{Tcf} 7 \mathrm{~L} 2^{\text {fl/fl }}$ mutants at E15. a-c Hematoxylin-eosin staining of coronal sections from controls, Tcf7L2 single and Tcf7L1/Tcf7L2 double mutants. d-f Pax6 and Ctip2 double immunofluorescence with DAPI showing RGC marker Pax6. d'-f'Ctip2 immunofluorescence showing neuronal marker Ctip2 in the cortical plate (CP) at E15 in Tcf7L1/Tcf7L2 double mutants. g-i Tuj1 and Tbr2 double immunofluorescence counterstained with DAPI showing the cortical plate and intermediate neuronal progenitors in the SVZ at E15. g'-i'a higher magnification of Tbr2+ cells in the SVZ. . j-I PH3 and ZO1 double immunofluorescence with DAPI showing normal adherens junctions and normally dividing $\mathrm{PH}^{+}$progenitors at E15. j'-l'PH3 and ZO1 double immunofluorescence without DAPI. (JPG 2237 kb)

\section{Abbreviations}

Cp: Choroid plexus; CP: Cortical plate; Cx: Cortex; DG: Dentate gyrus; DT: Dorsal thalamus; GE: Ganglionic eminences; Hi: Hippocampus; IPC: Intermediate progenitor cells; LV: Lateral ventricle; RGC: Radial glial cells; SVZ: Subventricular zone; VZ: Ventricular zone

\section{Acknowledgements}

We thank to K. Kovacova, J. Lachova for excellent technical support and to S. Piccolo for providing BAT-Gal mice. We acknowledge the Light Microscopy Core Facility, IMG ASCR, Prague, Czech Republic, supported by grants (Czech-Bioimaging - MEYS LM2015062), 'Centre for Model Organisms' OPPK (CZ.2.16/3.1.00/21547) and 'Biomodels for Health (LO1419). We further thank the animal facility at Czech Centre for Phenogenomics (CCP, project no. LM2015040)' and OP RDI CZ.1.05/2.1.00/19.0395 (Higher quality and capacity for transgenic models).

\section{Funding}

This work was supported by Czech Science Foundation, grant P305/12/2042 (OC, JM, OM, material and parts of salaries), grant 18-00514S (OM, ZK material and parts of salaries), grant 15-25100S (VK, material).

\section{Availability of data and materials}

The datasets used and/or analyzed during the current study are available from the corresponding author on request.

\section{Authors' contributions}

OC performed experiments and interpreted the data, acquisition of data for Figs 1, 2 and 3. JM performed experiments and interpreted the data, acquisition of data for Additional file 1: Figure S1, Additional file 2: Figure S2. ZK and VK designed experiments and conception, provided material including transgenic mouse strains and critically read manuscript. OM designed and performed experiments, acquisition of data for Fig. 4, and wrote the manuscript. All authors read and approved the final manuscript.

\section{Ethics approval}

All procedures involving experimental animals were approved by the Institutional Committee for Animal Care and Use (permission \#PP-071/2011). This work did not include human subjects.

\section{Competing interests}

The authors declare that they have no competing interests.

\section{Publisher's Note}

Springer Nature remains neutral with regard to jurisdictional claims in published maps and institutional affiliations.

\section{Author details}

${ }^{1}$ Institute of Molecular Genetics of the Czech Academy of Sciences, Vídeňská 1084, 14200 Prague, Czech Republic. ${ }^{2}$ Institute of Experimental Medicine of the Czech Academy of Sciences, Vídeňská 1084, 14200 Prague, Czech Republic. ${ }^{3}$ Present address: Department of Biosciences and Nutrition, Karolinska Institutet, SE-14183 Huddinge, Sweden. 'Laboratory of Eye Biology, Division BIOCEV, Institute of Molecular Genetics of the Czech Academy of Sciences, Prumyslova 595, Vestec, Czech Republic. 
Received: 21 February 2018 Accepted: 26 April 2018

Published online: 11 May 2018

\section{References}

1. Rash BG, Grove EA. Area and layer patterning in the developing cerebral cortex. Curr Opin Neurobiol. 2006;16:25-34.

2. Kriegstein AR, Noctor SC. Patterns of neuronal migration in the embryonic cortex. Trends Neurosci. 2004;27:392-9.

3. Noctor SC, et al. Cortical neurons arise in symmetric and asymmetric division zones and migrate through specific phases. Nat Neurosci. 2004;7:136-44

4. Noctor SC, Martinez-Cerdeno V, Kriegstein AR. Distinct behaviors of neural stem and progenitor cells underlie cortical neurogenesis. J Comp Neurol. 2008;508:28-44.

5. Noctor SC, Martinez-Cerdeno V, Kriegstein AR. Neural stem and progenitor cells in cortical development. Novartis Found Symp. 2007;288:59-73.

6. Chenn A, Walsh CA. Regulation of cerebral cortical size by control of cell cycle exit in neural precursors. Science. 2002;297:365-9.

7. Backman M, et al. Effects of canonical Wnt signaling on dorso-ventral specification of the mouse telencephalon. Dev Biol. 2005;279:155-68.

8. Machon $\mathrm{O}$, et al. Role of beta-catenin in the developing cortical and hippocampal neuroepithelium. Neuroscience. 2003;122:129-43.

9. Munji RN, et al. Wnt signaling regulates neuronal differentiation of cortical intermediate progenitors. J Neurosci. 2011;31:1676-87.

10. Hirabayashi Y, Gotoh Y. Stage-dependent fate determination of neural precursor cells in mouse forebrain. Neurosci Res. 2005:51:331-6.

11. Hirabayashi Y, et al. The Wnt/beta-catenin pathway directs neuronal differentiation of cortical neural precursor cells. Development. 2004;131:2791-801.

12. Harrison-Uy SJ, Pleasure SJ. Wnt signaling and forebrain development. Cold Spring Harb Perspect Biol. 2012;4:a008094.

13. Draganova $\mathrm{K}$, et al. Wnt/beta-catenin signaling regulates sequential fate decisions of murine cortical precursor cells. Stem Cells. 2015;33:170-82.

14. Hrckulak D, et al. TCF/LEF transcription factors: an update from the internet resources. Cancers (Basel). 2016;8:70.

15. Moreira $S$, et al. A single TCF transcription factor, regardless of its activation capacity, is sufficient for effective Trilineage differentiation of ESCs. Cell Rep. 2017;20:2424-38.

16. Galceran J, et al. Hippocampus development and generation of dentate gyrus granule cells is regulated by LEF1. Development. 2000;127:469-82.

17. Galceran J, et al. Wnt3a-/--like phenotype and limb deficiency in Lef1(-/-)Tcf1(-/-) mice. Genes Dev. 1999;13:709-17.

18. Vendrell $V$, et al. Gene expression analysis of canonical Wnt pathway transcriptional regulators during early morphogenesis of the facial region in the mouse embryo. Gene Expr Patterns. 2009;9:296-305.

19. Maretto $\mathrm{S}$, et al. Mapping Wnt/beta -catenin signaling during mouse development and in colorectal tumors. PNAS. 2003;100:3299.

20. Machon O, et al. A dynamic gradient of Wnt signaling controls initiation of neurogenesis in the mammalian cortex and cellular specification in the hippocampus. Dev Biol. 2007;311:223-37.

21. Grove EA. Neuroscience. Organizing the source of memory. Science. 2008;319:288-9.

22. van den Bout CJ, et al. The mouse enhancer element D6 directs Cre recombinase activity in the neocortex and the hippocampus. Mech Dev. 2002;110:179-82.

23. Masek J, et al. Tcf7l1 protects the anterior neural fold from adopting the neural crest fate. Development. 2016;143:2206-16.

24. Zechner D, et al. Beta -catenin signals regulate cell growth and the balance between progenitor cell expansion and differentiation in the nervous system. Dev Biol. 2003;258:406-18.

25. Korinek $V$, et al. Depletion of epithelial stem-cell compartments in the small intestine of mice lacking Tcf-4. Nat Genet. 1998;19:379-83.

26. Lien WH, Fuchs E. Wnt some lose some: transcriptional governance of stem cells by Wnt/beta-catenin signaling. Genes Dev. 2014;28:1517-32.

27. Lien $\mathrm{WH}$, et al. In vivo transcriptional governance of hair follicle stem cells by canonical Wnt regulators. Nat Cell Biol. 2014;16:179-90.

28. Wallmen B, Schrempp M, Hecht A. Intrinsic properties of Tcf1 and Tcf4 splice variants determine cell-type-specific Wnt/beta-catenin target gene expression. Nucleic Acids Res. 2012;40:9455-69.

29. Nguyen $\mathrm{H}$, et al. Tcf3 and Tcf4 are essential for long-term homeostasis of skin epithelia. Nat Genet. 2009;41:1068-75.
30. Gregorieff A, Grosschedl R, Clevers $H$. Hindgut defects and transformation of the gastro-intestinal tract in Tcf4(-/-)/Tcf1(-/-) embryos. EMBO J. 2004;23:1825-33.

31. Kuwahara A, et al. Tcf3 represses Wnt-beta-catenin signaling and maintains neural stem cell population during neocortical development. PLoS One. 2014;9:e94408

32. Ohtsuka T, et al. Gene expression profiling of neural stem cells and identification of regulators of neural differentiation during cortical development. Stem Cells. 2011;29:1817-28.

33. Cole MF, et al. Tcf3 is an integral component of the core regulatory circuitry of embryonic stem cells. Genes Dev. 2008;22:746-55.

34. Soriano P. Generalized lacZ expression with the ROSA26 Cre reporter strain. Nat Genet. 1999;21:70-1.

\section{Ready to submit your research? Choose BMC and benefit from:}

- fast, convenient online submission

- thorough peer review by experienced researchers in your field

- rapid publication on acceptance

- support for research data, including large and complex data types

- gold Open Access which fosters wider collaboration and increased citations

- maximum visibility for your research: over $100 \mathrm{M}$ website views per year

At BMC, research is always in progress.

Learn more biomedcentral.com/submissions 